\title{
Replication Based on Role Concept for Multi-agent Systems
}

\author{
Sebnem Bora Oguz Dikenelli \\ Computer Engineering Department \\ Ege University, Izmir, Turkey \\ sebnem.bora,oguz.dikenelli@ege.edu.tr
}

\begin{abstract}
Replication is widely used to improve fault tolerance in distributed and multi-agent systems. In this paper, we present a different point of view on replication in multi-agent systems. The approach we propose is based on role concept. We define a specific "fault tolerant role" which encapsulates all behaviors related to replication-based fault tolerance in this work. Our strategy is mainly focused on replicating instances of critical roles in the agent organization. However, while doing this, we simply transfer the critical role and the fault tolerant role to appropriate agents. Here, the fault tolerant role is responsible for coordination between replicated role instances (replicas). Moreover, our approach is flexible in terms of fault tolerance since it is possible to easily modify existing behaviors of the "fault tolerant" role, remove some of its behaviors, or include new behaviors to it due to its characteristic architecture.
\end{abstract}

\section{Introduction}

Multi-agent systems have recently been widely employed in solving problems faced in distributed and dynamic environments. As distributed systems, multi-agent systems (MAS) are vulnerable to failures resulting from the system crash or shortages of system resources, slow-downs or breakdowns of communication links, and errors in programming. Consequently, a fault may spread throughout a multi-agent system, and cause a degradation of the system performance and even the multi-agent system to fail. Therefore, it seems that fault tolerance is a necessary paradigm that must be inserted to the multi-agent development environment.

In most cases, replication-based techniques are employed in multi-agent systems to achieve fault tolerance such as explained in $[6,7,8,9,10,11,12,19,20]$. In these approaches, they replicate the agent itself by applying standard techniques used in distributed systems, although agents are more sophisticated and interactive entities.

In multi-agent organizations [18, 17], agents enact their roles and naturally, they act and communicate under their roles. Thus this affects their criticalities in the multiagent organization. For instance, a manager role can be considered more critical role than a janitor role in a factory since the manager role has critical responsibilities in terms of the organization such as supervising and taking charge of the activities and 
productivity of their workers, making organizational decisions and handling a variety of problems that arise on a daily basis. There are some other responsibilities of managers such as goal setting, planning, and organizing; evaluating and analyzing; and providing satisfaction among the staff and the customers. If an agent enacts critical roles for the organization, it then possesses all of its roles' goals and features including its roles' criticalities. The failure of this agent affects the organization that it belongs to. To apply fault tolerance in an organization, replication is the technique we need to apply. However, we should not replicate the agent itself.

To make easy to understand why agent replication is not appropriate for fault tolerance, let us give an example. We assume that there is an agent that enacts a critical role $\mathrm{r} 1$ and we replicate this agent due to enacting $\mathrm{r} 1$. Let's say there are two replicated agents with role $\mathrm{r} 1$ in the organization. Then, the original agent with role $\mathrm{r} 1$ wants to enact role $\mathrm{r} 2$. We have to answer questions such as "Should the second replicated agent take up the role r2?", "How many agents do we replicate for role r2?" etc. We can increase the number of questions while expanding this scenario. Thus, we understand that agent replication is a naïve way to improve fault tolerance in MAS organization and we have to find out a new way of replication in MAS.

In this paper, we present a new approach for replication in MAS. Our method is based on role concept. Therefore, we define a specific "fault tolerant role" which encapsulates all behaviors related to replication-based fault tolerance. The fault tolerant role is assigned to the agents that enact critical roles which are identified at the design time. One of the responsibilities of the fault tolerant role is to create new instances of critical roles. Therefore, the critical and "fault tolerant" roles are transferred to appropriate agents that will enact these roles. Since the "fault tolerant" role is also responsible for ensuring coordination among replicated instances of critical roles it should be enacted by the agents that enact the critical roles.

The remainder of this paper is structured as follows: Section 2 presents the context of the work; Section 3 presents the "fault tolerant" role and how to implement rolebased replication in multi-agent organizations; Section 4 describes how to determine criticalities of roles; Section 5 gives a case study; Section 6 is a review of related work on fault tolerance in multi-agent systems and finally Section 7 gives the conclusion.

\section{Context of This Work}

In this section, we describe the proposed architecture for role-based replication. We use the model shown in Figure 1 to describe role-based replication.

In this model, a role is one of the basic components of a multi-agent organization and it is seen as performing a goal within the organization to serve some purpose. A role has its goals, plans (a partially-ordered sequence of primitive tasks), and ontological knowledge. The concept of a goal allows specifying objectives as the desired final state to be reached. Agents are active entities that enact role(s) and achieve goals of their own roles by executing its roles' plans. A role enacting agent uses its role's knowledge via its role's plans to achieve its role's goal. For instance, as explained in [17], in order to effectively play the role of cook in a restaurant, a person 
has to follow work instructions, use the tools required for the role to transform the ingredients as required, and communicate with other role players. In this model, we assume that agents have their own goals, knowledge, and capabilities to enact some specific roles [26].

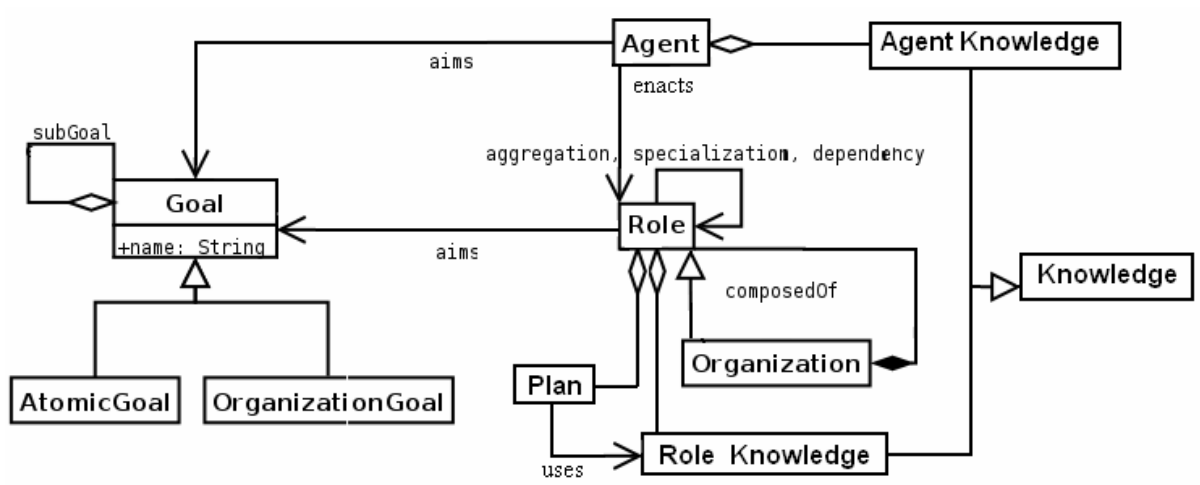

Fig. 1. The Model We Use to Describe the Proposed Architecture

If one aims to improve fault tolerance for this model, he has to identify new goals coming from fault tolerance requirements, plans to achieve these goals, and reusable services that can be used by these plans. However, we first need to present some basic concepts. A system is said to fail when it cannot meet its specification. Therefore, a deviation from the specification is considered a failure. A fault is defined as a defect that may lead to a failure. Whenever a failure occurs, a fault-tolerant system should continue to operate in a degraded fashion while repairs are being made, i.e. system should tolerate faults and continue to operate in the presence of faults. Our goal in multi-agent systems design is to construct a multi-agent organization in such a way that it can automatically recover from partial failures without seriously affecting the overall performance.

If we aim to construct a fault tolerant system, we have to answer what sort of partial failures can degrade the system's performance. Since the role concept is very important aspect of an organization in this model, the roles enacted by the agents of the organization must be avoided to be failed. We list below under what circumstances the role is failed:

1. The agent that enacts this role is crashed.

2. Some parts of communication links are crashed. Since the agent cannot communicate with agents then it will not achieve its role's goal in a timely manner.

3. Some resources can not be accessed. Since a role has rights to access resources which belong to environment.

4. There are some conflicts in the knowledge bases of the different roles of the agent. Since the agent can enact more than one role, the knowledge bases of the roles enacted by the agent can have some rules that can seriously contradict each other. 
5. The agent does not have all the plans of the role it enacts.

All these situations contribute to a failure state. The failure model defines the ways in which failure may occur to provide an understanding of the effects of failures. In this work, we focus on the fail-silent model where the considered system allows only crash failures [27]. Therefore, we'll not focus on handling with items 3, 4 and 5 in this paper.

Since replication is a key technique to achieve fault tolerance in distributed and dynamic environments, we use this technique to avoid failures resulting from the failed roles. We should replicate the instances of critical roles to mask the failed roles from the organization. To achieve this, we should find agents that can take up the critical role. Then, we should transfer the critical role that encapsulates its goals, plans and knowledge to the agents.

To apply role-based replication in a multi-agent organization, there must be several services such as the replication service, the group communication service, the failure detection, and the membership service. In general, the replication service is responsible for creating new replicas and destroying some existing replicas and applying active and passive replication approaches. Active and passive approaches mainly focus on coordination between the replicas [13].

In addition to coordination requirements, the replication degree, which means the number of replicas, is a critical concept for applying fault tolerance policies based on replication. The problem is how the system will decide on the number of replicas at runtime. The replication degree can be identified adaptively or statically. In a static fault tolerance policy, this number is set to the number defined by a programmer at initialization. In an adaptive fault tolerance policy, the number of replicas of the group is determined based on resources of the system.

We have some further constraints on replication-based fault tolerance in MAS. Since the criticalities of roles may change dynamically during the system's operation and the available resources are not infinite, the number of replicas of a role instance must be dynamically updated based on the criticalities of the role as well. In order to insert fault tolerant structures to a MAS and to cope with all these constraints derived from replication, we propose a specific role called "fault tolerant role". Next, we present role-based replication.

\section{Role-Based Replication}

When the agent enacts a role, it behaves under this role. An agent can enact different roles from moment to moment. Each agent can enact several roles at one time beyond the basic role of AgentId. Every agent enacts AgentId role and each agent has at least that role at all times $[22,18]$. For role changing, several operations such as "classify", "declassify", "reclassify", "activate", "suspend" and "shift" are introduced by Odell et al. [18]. These operations are primitive operations and come with the basic role of AgentId.

For the role-based replication, there must be several instances of a critical role (replicas). In the case of failure of one of replicas, there will be another replica to 
achieve its critical goal in the organization. In this work, we introduce the "fault tolerant" role which has some goals coming from replication-based fault tolerance requirements, plans to achieve these goals, and reusable services that can be used by these plans. This role must be enacted by all agents that enact the critical role or any role that we want it not to be crashed, since the fault tolerant role is responsible for ensuring coordination between replicas. This role provides consistency between replicas.

To simplify the understanding of the role-based replication process, we rediscuss the example given in [18] from the fault tolerance perspective. Figure 2 portrays the "Nancy" agent being classified the secretary of an executive director in the Organization B. The organization decides that her role in the company (Organization B) is very critical. Therefore, she'll enact the fault tolerant role and have some replicas. When she cannot work in her company due to some reasons, there will be nothing to worry about the organization's operation. In this case, replicas can take over her responsibilities.

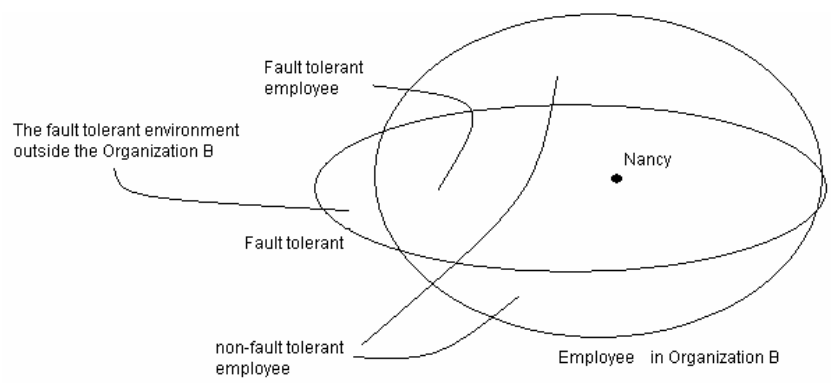

Fig. 2. Dynamic Classification in terms of Fault Tolerance (1)

Figure 3 illustrates the "Susan" agent being classified the co-secretary of an executive director in the Organization B. As defined in [18], the "Classify" operation simply adds the transferred role to other roles' group of the agent. She is the replica for Nancy's role and she also enacts the fault tolerant role. Fault tolerant role helps providing coordination between Nancy and Susan. Thus Susan has the knowledge, goal, and plan of the role of Nancy as well.

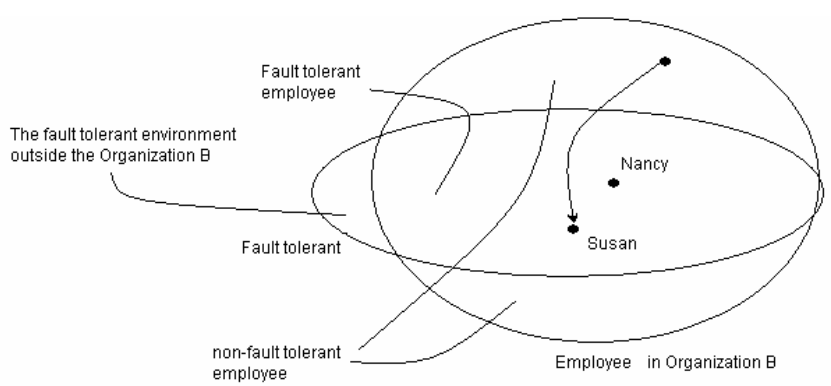

Fig. 3. Dynamic Classification in terms of Fault Tolerance (2) 
In addition to operations introduced by Odell et al. [18] to deal with dynamic role assignment, we introduce some more operations: "Evaluate", "Find", "Transfer". These operations are needed due to the role-based replication process and we assume that they also come from the basic role of AgentId.

For role-based replication, the first operation is to evaluate criticalities of roles (RoleCriticality), if the adaptive fault tolerance policy is preferred to apply to the organization. The criticality of a role is the measure of the potential impact of the failure of that role on the failure of the organization. For this purpose, each agent that enacts the "fault tolerant" role executes a periodic plan called "calculation of criticalities of roles". In this plan, the agent periodically invokes the "Evaluate" operation and calculates the criticality of the role that it enacts. The value of RoleCriticality is then used to compute the number of replicas for the critical role.

For role-based replication, the next operation is to find suitable agents that can take the critical role for the replication process, if necessary. This is currently being investigated [25], and will not be considered in this paper. We suppose that there are lightly loaded agents that we can transfer the critical role to them. If there is no such an agent, we have to create new agents that can enact the critical role. After selecting or creating an agent, we have to transfer the critical role as an entity including its knowledge, its goal and plan libraries (behaviors) to the agent that will enact a given role. Therefore, "Transfer" operation is invoked. To coordinate the replicas, we also transfer the "fault tolerant" role to the agents that enact the critical role. The fault tolerant role associates with only the critical role for the coordination purpose and encapsulates fault tolerance related behaviors and provides interactions between replicas. The original agent that enacts critical role, takes up the fault tolerant role when we decide on applying role-based replication. After transferring the critical role and the "fault tolerant" role to the agents, we have to classify these roles. Thus agent enacts the critical role and the fault tolerant role (multiple classification) when it is classified.

While the agent enacts a role, this role can be either activated or suspended. To activate our new critical and fault tolerant roles, we have to perform the "Activate" operation.

Fault tolerant role is very important for improving fault tolerance in an organization since agents that enact the critical role behave under their fault tolerant roles to provide coordination between replicas. In an organization, the leader (that originally enacts the critical role) may quit the organization or it may be removed in the case of if the agent enacting this role is failed due to some reasons. Then, a replica can take over. It has all the necessary stuff such as knowledge, plans to achieve its goal. Next, we "ll describe the "fault tolerant" role in detail.

\subsection{The Fault Tolerant Role}

Figure 4 shows a goal model for a simple fault tolerant organization in our approach. In this model, the organization includes some roles such as the fault tolerant role, a role which is critical, and some other roles. To perform role-based replication, the fault tolerant role has the goals such as "multicast to the group", "detect failures", 
"elect a new leader", "monitor criticalities of roles", and "adjust the number of replicas" goals. In order to achieve these identified goals, we implement their plans and the reusable services that can be used by these plans [11].

One of the reusable services is the membership service. It maintains a list of replicas of a replica group in the knowledge base of each member of the replica group. The membership service associates with a failure detection service to reach a decision about the group's membership. The output of the membership service is called a "view" $[13,14]$. The reliable multicast services deliver messages to the current view members.

Failure detection is one of the crucial aspects of any fault tolerant system to recognize a faulty component of the system. In our approach, "sendAlive" and "receiveAlive" are two plans that achieve the "detect failures" goal. In the "sendAlive" plan, each replica in the replica group periodically exchanges heartbeats. A heartbeat message is an "I am alive" message prepared for informing that the sending replica is safe.

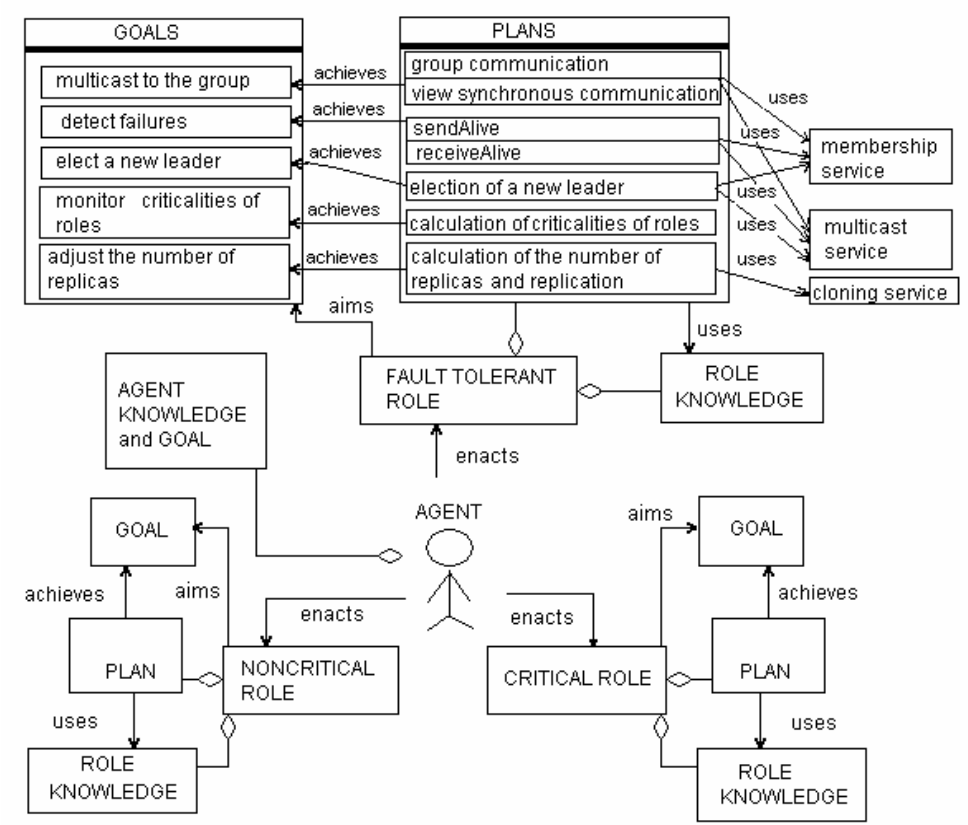

Fig. 4. A Goal Model for a Simple Fault Tolerant Organization

Once a heartbeat message has been received by all the replicas, then the "receiveAlive" plan is executed. In this plan, each agent waits for a new heartbeat message from the same agent that sent the previous heartbeat message. If a new heartbeat message is not received during timeout, that replica is added to a suspect list. If all replicas detect that replica on timeout, they place the replica in question on their suspect lists. In the case of recurrence of the same situation, the replica is deleted from the membership lists. Timeout values are set before application starts. However, 
timeout values are adapted according to observed response times. For instance, if a replica or the connection is very slow, then the timeout value will be increased to account for disparity; however, this may not prevent to mark a replica suspect falsely in all cases.

Coordination between replicas is supported by a group communication service which provides multi-point-to-multi-point communication. We assume that the MAS development framework also supports multicasting. This feature is used to implement the group communication service. However, this basic multicasting mechanism only delivers requests in an arbitrary order. An ordering guarantee in multicasting must be supported to develop fault tolerant systems because consistency and coordination between the replicas is constructed by performing incoming requests in order. The ordering scheme is implemented in the "group communication" plan which achieves the "multicast to the group" goal. In this plan, a group-specific ascending number is assigned to a new message whenever it is received from another agent, and then this message is multicast to the replicas in the membership lists in order to implement the semi-active replication strategy. In semi-active replication strategy, replicas are organized in a group, and all replicas execute incoming requests. One of the agent that enacts the critical role is designated as the primary (the leader) and responsible for providing responses. Therefore, consistency between replicas is ensured by processing each message.

In the passive replication strategy, replicas are also organized in a group and primary one responds to client requests. However, primary periodically updates replicas' states. If primary fails, one replica can be elected as a primary. In this work, the passive replication strategy uses the primary to order the messages as the semiactive replication strategy does, but requires a mechanism to order the views. Therefore, we have implemented the view synchronous multicast for the passive replication strategy. The inconsistency between replicas is avoided if, whenever the primary sends the update message to its replicas, either all or none of the replicas receive the message (atomicity) in the context of a dynamic membership [23]. View synchronous multicast is also implemented in the "view synchronous communication" plan. The implementation consists in delivering multiple, independent, a batch of messages between view changes. A view change occurs when a replica wants to join or leave the group, or a replica fails. In every view change, a message containing the composition of the view of the group is multicast to the group members. If the primary of the group fails, and a new view is defined, either all the replicas in new view, or none of them consider the last message of the primary. Therefore, consistency in the group will be ensured.

The replication service is the most important part of the replication-based fault tolerance approaches. To implement the replication service in our approach, the "adjust the number of replicas" goal must be achieved. The "calculation of the number of replicas and replication" plan achieves this goal by using the cloning service. In this plan, the replication degree of the group is calculated by using the value of the criticality of a critical role and the number of resources. If the replication is needed according to the result of the replication degree, then the cloning $[11,15,16]$ service is used for replication of new replicas.

The critical roles must be identified to replicate new instances of the critical roles. To identify critical roles, the number of messages received by an agent for each role is 
monitored to collect some data to calculate the role criticalities. The collected data is then evaluated to take a decision on the role criticalities. The fault tolerant role is responsible for monitoring the roles and evaluating the criticalities of roles. Therefore, the fault tolerant role has the "monitor criticalities of roles" goal and the "calculation criticalities of roles" plan to achieve that goal. The criteria to be a critical role will be given in the next section.

In the proposed architecture, each role instance can be replicated many times and with different replication strategies. Each replication group has only one leader which coordinates the replica group and communicates with the other replicas. When the leader fails, a replica is selected as a new leader in the replica group. Therefore, the fault tolerant role has the "elect a new leader" goal and the "election of a new leader" plan to achieve this goal.

Next, we describe what makes an agent critical and the criticality of the role.

\section{Criticality of the Role}

In order to achieve effective fault handling, we have to estimate the critical roles of the organization, since the criticality of a role is the measure of the potential impact of the failure of that role on the failure of the organization. There are two cases that we must distinguish: 1) the role's criticality is static and 2) the role's criticality is dynamic. In the first case, multi-agent systems have static organization structures. Therefore, critical roles can be identified and then replication is performed by the programmer before run time.

In the second case, the role criticality cannot be determined before run time since the multi-agent systems may have dynamic organization structures. Therefore, critical roles can be dynamically evaluated at run-time. It can be done by using some prior input, or it can be done by using an observation module that collects the data $[10,11]$. We can use some metrics for dynamically estimating and updating role criticalities in the organization. Within a MAS organization, the roles designate responsibilities of agents. Each role has a different impact on the operation of organization. For instance, Colman and Han distinguish types of roles in an organization: functional roles, operational-management roles and organizational management roles [17]. The concept of role captures the importance of an agent in an organization, and its dependencies to other agents.

The dependency of other roles on a specific role is another metric that can show the role's criticality. If a critical role somehow fails, the other roles, which rely on the critical role, will struggle to achieve their individual goals. The dependency on a role is derived from the number and performatives of the messages received. Guessoum et al. distinguishes six classes of performatives [12]. They also describe the influence of a message on its receiver by using symbolic values such as low, medium, high. According to this classification, we only consider messages (containing Class 1 type of performatives) [12] with a high value of influence on their receivers to determine the criticality of a role. Therefore, messages, to be considered in terms of role's criticality in our approach, contain performatives such as request, request-whenever, query-if, query-ref, subscribe. 
In this work, we set a period for the organization at the system initialization. This period is called the sampling period $\mathrm{T}$ and actually defined over a time window $((\mathrm{k}-$ 1) T, kT), where k is the sampling instant. Considering both metrics, the criticality of a role and the number of replicas are calculated in the "calculation of criticalities of roles" and "calculation of the number of replicas, and replication" plans. The criticality of a role can be defined by the following equations:

RoleCriticality $(k)=a *$ role $(k)+b *$ Ratio_req $(k)(1)$

Ratio_req $(k)$ : The change in the number of messages sent to an agent for a specific role to the average number of messages over a number of periods;

$a, b$ : Coefficients for contributions of the weight of the role and the number of requests to the RoleCriticality.

role $(k)$ : The value corresponding to the weight of the role at the kth sampling instant. The weights of the roles are explicitly defined in the role ontology before the program starts. Consider weight of each role as a numerical value within the interval $(1,10)$.

After we have computed the criticality of each role, we can compute the number of replicas (no_replicas) of a critical role as follows:

$n o \_$replicas $(k+1)=\operatorname{rounded}(r m+R M *$ RoleCriticality $(k))$

$r m$ : the minimum number of replicas that is defined by programmer before an application starts.

$R M$ : The available resources that define the maximum number of possible simultaneous replicas. Then, the number of replicas (no_replicas) is used to control and update replication for each role in the next sampling period.

$R . C=n o \_r e p l i c a s(k+1)$-no_replicas $(k)$

where R.C is the number of replicas that must be replicated if $R$. $C$ is a positive integer or the number of replicas that must be killed if $R$. $C$ is a negative integer. In the next we'll give the case study.

\section{Case Study}

Our fault tolerance approach presented in this paper has been implemented as a case study by using SEAGENT Multi-Agent framework [1, 24]. For the case study, we designed an agent system which includes some specific agents which enact the "library assistant" role (library assistant agent), or enact the "user assistant" role for querying library assistant agents. The library assistant agent manages a library and holds the library knowledge using the library ontology. Instances of this ontology hold the properties of books including name, ISBN, authors' names and keywords of the books. In our case study, each user assistant agent directly sends a book request to library assistant agents. The library assistant agent has only one plan that matches the request to the book ontology instance(s) and returns the matched books' descriptions within a FIPA-ACL message [2]. When the user assistant agent receives responses from the library assistant agents, it selects a library based on the responses and presents the result to the user. In this case study, the user assistant agents depend on the library assistant agents. Therefore, each library assistant agent is a single of point of failure. Since the library assistant agent is a critical agent for the system's 
operation, it must be initialized as a fault tolerant agent. In order to become a fault tolerant agent, the library assistant agent should also enact the "fault tolerant" role. The goal model of this scenario is shown in Figure 5.

In this goal model, three types of roles are given: The library assistant role, the user assistant role and the fault tolerant role. The user assistant role has the "send a query" goal to query the library assistant agent and the "present the result to the user" goal to present the results that the user waits for. The library assistant role has the "process the query" goal to process the requests and return the results. The "fault tolerant" role has the "send alive" and "receive alive" goals to implement the failure detector, the "adjust the number of replicas" goal to handle the replication process, the "elect a new leader" goal to elect a new leader when the leader fails, the "monitor criticalities of roles" goal to calculate the criticalities of roles, and the "multicast to the group" goal to implement group communication.
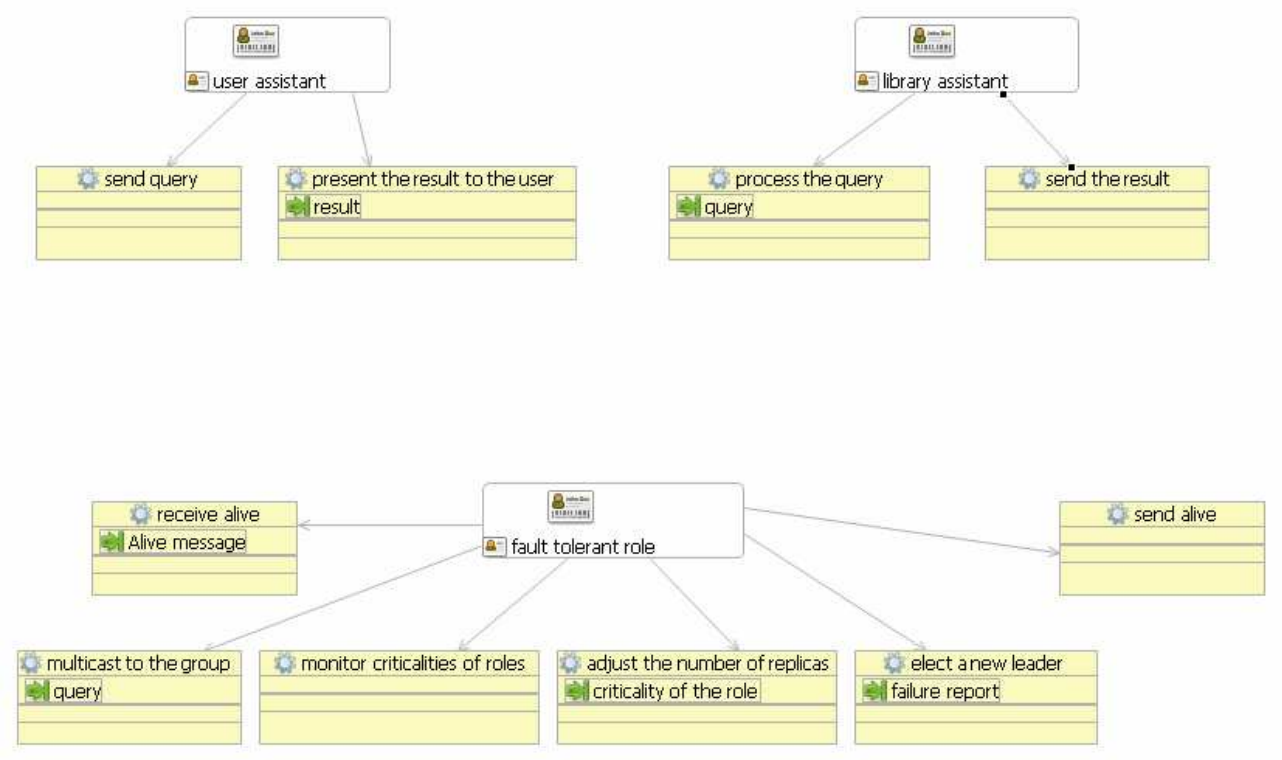

Fig. 5. A Goal Model for the Fault Tolerant Library System

When we decide that the library assistant agent needs to be fault tolerant, it invokes a "classify" operation for the "fault tolerant" role, it then enacts the "fault tolerant" role. Then, it invokes a "find" operation to search for a replica candidate residing on another host. When it finds an agent to take up the "library assistant" role, it then transfers the "library assistant" and "fault tolerant" roles including their plan libraries. These all operations are performed in the "calculation of the number of replicas and replication" plan to achieve the "adjust the number of replicas" goal. When the agent on the other host classifies both roles, the agent enacts the "library assistant" role and activates it. When the agent enacts the critical role and the fault tolerant role, it and its leader periodically execute the "sendAlive" plan to implement the heartbeat mechanism of the failure detection mechanism. 
Let us assume that the user assistant agent sends a query to the leader library assistant agent and apply the semi-active replication scheme. When the leader library assistant receives this query, it automatically assigns a number to this query and sends it to itself and its replica by executing the "group communication" plan to achieve group communication goal. When the library assistant agents receive this query, they process the query. The leader library assistant agent returns the result to the user assistant agent.

\subsection{Evaluation of the Approach}

To evaluate our approach, we implemented a test bed for the scenario mentioned above. In this test bed, we suppose that multi-agent organizations are static structures and the criticalities of roles do not change over time in such a system. Therefore, we identify that the "library assistant" role to be replicated and define the replication degree for this role before program starts. The library assistant role and some other roles having simple plans are assigned to an agent. In the first case, we employ the agent replication approach in the test bed. According to the agent replication approach, the agent is replicated due to having the critical "library assistant" role. Therefore, we implemented a test bed consisting of a library assistant agent leader, its replicas (agents) in the number range from 3 to 23, and the user assistant agent that queries the library assistant agent. In this test bed, another agent also sends requests to the library assistant agents due to their other roles. Since the agent itself is replicated, then all roles enacted by the leader are also enacted by the replica agents. In the second case, we employ the role-based replication approach in the test bed. According to our approach, the critical role and the fault tolerant role are assigned (transferred) to the agents having less workload. In this case, the test bed consists of the replica instances of the library assistant role (together with the fault tolerant role) in the number range from 3 to 23 , the user assistant agent, and the leader. The user assistant agent periodically queries the agent that enacts the "library assistant" role. The number of queries sent to the agent that enacts "library assistant" role is equal to 60 for both cases. Also in the second case, the agents that enact "library assistant role" are queried by another agent for their other roles to cause workload on them. In order to see the costs of role-based replication and agent replication, the response times for queries are measured in both cases. The response time is the time it takes the user assistant agent to receive the last reply from a leader after sending its requests to the leader. In this test, we try to observe the effect of role-based replication to the overall system performance.

The agent system is implemented in the SEAGENT platform and Java Version 1.5.0. The tests are performed on two computers with Intel P4 running at $1.5 \mathrm{GHz}$ and 2GB of RAM, running Windows XP. We distribute the agents to two computers and run each test ten times.

The results of the tests are illustrated in Fig. 6. The results show the average response times of the systems applying the agent replication and role-based replication approaches, as the number of replicas increases. As seen from the figure, the slopes of response times of both systems increase linearly with the number of agents in an organization. The increase in response time is expected, since it is caused 
by the implementation of the system on two machines. Moreover, the number of the exchanged messages increases with the number of replicas due to the multicasting of request and heartbeat messages. However, the average response times of the system applying the agent replication are much higher than the average response times of the system applying role-based replication. This result was also expected, since the agent is replicated with all its roles due to having a critical role into an agent replica group. The leader of the group multicasts requests to its group members.

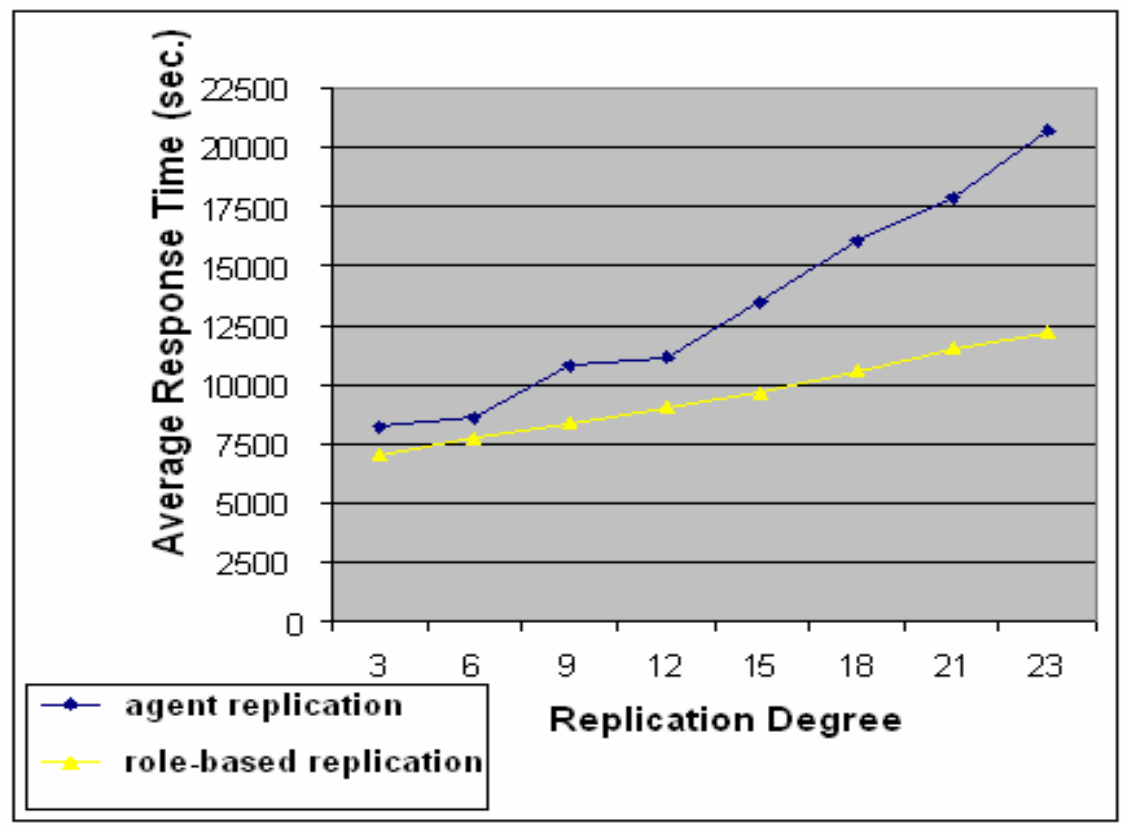

Fig. 6. The Test Results

\section{Related Works}

Several approaches to fault-tolerance in MAS are documented in the literature; each focuses on different aspects of fault-tolerance. Kumar et al. present a methodology than can be used to specify robust brokered architectures with capability of recovering from broker failures [3]. Their methodology is based on the theory of teamwork. In their work, brokers are organized hierarchically in teams. Brokers in teams exchange information between them and maintain communications between agents. Their approach can be only used for recovering from broker failures.

Klein proposes an approach based on a shared exception handling service that is plugged into existing agent systems [4]. This service monitors the overall progress of a multi-agent system. When a new agent is created, the "new agent registration" agent takes a description of its normative behavior and creates sentinels to look for the evidence of dysfunctional behavior. When a sentinel detects such faulty symptoms, this information is sent to a "diagnosis" agent that produces a set of candidate 
diagnoses. These are sent to the resolution agent that defines a resolution plan to take corrective actions.

Hagg uses sentinel agents to guard specific functions or to guard against specific states in the society of agents. The sentinel interacts with other agents using semantic addressing. Thus, it can build models of other agents by monitoring agent communication and by interaction. It can also use timers to detect crashed agents or communication link failures [5].

There are also well-known fault tolerance approaches based on replication techniques for multi agent systems. In order to increase fault tolerance and improve availability and reliability of MAS, Fedoruk and Deters implemented transparent replication via proxies [6]. The proxy as an interface handles all communication between replicas and other agents in the MAS. The proxy also controls execution in a replica group and state management of a replica group. Although this proxy approach handles fault tolerance issues in a multi-agent system, proxy itself is a single point of failure. There is no recovery mechanism introduced in this work when the proxy fails. They chose FIPA-OS agent toolkit as a platform for their implementation. Since FIPA-OS does not provide any replication mechanism, the replication server is implemented as a standard FIPA-OS agent. Moreover, this approach does not support the idea of changing fault tolerance policies at run-time. Therefore, replication is realized by a programmer before the application starts.

Guessoum et al. present an adaptive multi-agent architecture with both agent level and organization level adaptation [7, 8]. The organization's adaptation is based on the monitoring of the system's behavior. The architecture was implemented with the DIMA platform [9] and the DarX middleware [10]. In DarX, software components can be either replicated or un-replicated, and it is possible to change the replication strategy at run time. Although we also use the replication technique to implement fault tolerance policies within the organization, the main difference of our approach from this work is that our replication technique is based on role concept. In our replication technique, we do not replicate an agent due to its criticality. Because, we claim if an agent is critical in the organization, its role that it fills as a position is critical for the organization. Therefore, we transfer behavior libraries (plans) and knowledge of the critical role to an agent to become an instance of the critical role.

These approaches present useful solutions to the problem of fault tolerance in multi-agent systems. However, the entities used for handling this problem force a specific multi-agent organization and these approaches lack flexibility and reusability. On the other hand, in our case, fault tolerance requirements are identified and the behaviors that satisfy these requirements are encapsulated by the fault tolerant role. These behaviors can be used whenever we need to make an organization fault tolerant. Briefly, our approach provides flexibility and reusability to multi-agent organizations in terms of fault tolerance since it is possible to easily modify existing behaviors of the fault tolerant role, remove some of behaviors, or include new behaviors. 


\section{Conclusion}

In this paper, we presented a new approach to replication-based fault tolerance in MAS. We define a "fault tolerant" role that encapsulates all the related behaviors to replication-based fault tolerance such as replication, failure detection mechanism, group communication service, leader election, and monitoring. Therefore, the "fault tolerant" role is responsible for replicating instances of critical roles, coordination between critical role instances and satisfying all replication-based fault tolerance requirements.

Moreover, our approach is flexible in terms of fault tolerance since it is possible to easily modify existing behaviors of the "fault tolerant" role, remove some of its behaviors, or include new behaviors to it.

\section{References}

1. Dikenelli, O. et al. SEAGENT: A Platform for Developing Semantic Web Based Multi Agent Systems, The Fourth International Joint Conference on Autonomous Agents AAMAS05, 2005.

2. FIPA, FIPA Specifications, http://www.fipa.org

3. Kumar S., Cohen P. R, and Levesque H. J.: The adaptive agent architecture: Achieving faulttolerance using persistent broker teams. In Proceedings, Fourth International Conference on Multi-Agent Systems,July 2000.

4. Klein M. and Dallarocas C.: Exception handling in agent systems. Etzioni O., Muller J. P.. and Bradshaw J. M. editors, Proceedings of the Third International Conference on Agents (Agents'99) pages 62-68, Seattle,WA, 1999.

5. H“agg. S.: A sentinel approach to fault handling in multi-agent systems. In Proceedings of the second Australian Workshop on Distributed AI, in conjunction with the Fourth Pacific Rim International Conference on Artificial Intelligence (PRICAI'96), Cairns, Australia, August 1996.

6. Fedoruk A. and Deters R.: Improving fault-tolerance by replicating agents. In Proceedings of 1st International Joint Conference on Autonomous Agents and Multi-Agent Systems, Bologna, Italy, 2002.

7. Guessoum Z., Ziane M., Faci N.: Monitoring and organizational-level adaptation of multiagent systems. AAMAS'04, ACM, pp. 514-522, New York City, July 2004.

8. Guessoum Z., Briot J.-P., Charpentier Z., Aknine S., Marin O. and Sens P.: Dynamic and Adaptative Replication for Large-Scale Reliable Multi-Agent Systems, Proc. ICSE'02 First International Workshop on Software Engineering for Large-Scale Multi-Agent Systems (SELMAS'02), ACM, Orlando FL, U.S.A., May, 2002.

9. Guessoum Z. and. Briot J. P.: From active objects to autonomous agents. IEEE Concurrency, 7(3):68-76, 1999.

10. Guessoum Z., Briot J. P., Sens P., and Marin O.: Toward fault-tolerant multi-agent systems.In MAAMAW'2001, Annecy. France, 2001.

11. Bora, S., Dikenelli, O. Implementing A Multi Agent Organization That Changes Its Fault Tolerance Policy at Run-time. In: Proceedings of ESAW'05. Lecture Notes in Computer Science, Berlin, Germany, Springer Verlag, Volume 3963, pages 153-167, 2006. 
12. Guessoum Z., Faci N., and. Briot J. P.Adaptive Replication of Large-Scale MultiAgentSystems - Towards a Fault-Tolerant Multi-Agent Platform. Lecture Notes in Computer Science, Berlin, Germany, Volume 3914, pages 238-253, 2006.

13. Tanenbaum A. S. and van Steen M.: Distributed Systems: Principles and Paradigms, Prentice-Hall, 2002.

14. Chockler G. V., Keidar I., and Vitenberg R.: Group Communication Specifications: A Comprehensive Study, ACM Computing Surveys 33(4), pages 1-43, 2001

15. Shehory O., Sycara K., Chalasani P., and Jha S.: Agent Cloning: An Approach to Agent Mobility and Resource Allocation. IEEE Communications, Vol. 36, No. 7, pp. 58-67 1998.

16. Decker K., Sycara K., and Williamson M.: Cloning for Intelligent Adaptive Information Agents. In ATAL'97, LNAI, pp.63-75, Springer Verlag, 1997.

17. Colman, A. W. and J. Han,: Roles, Players, and Adaptable Organizations, Applied Ontology 2, 2007.

18. Odell J., Parunak H. V. D., Brueckner S., and Sauter J. A.: Changing roles: Dynamic role assignment. Journal of Object Technology, 2(5):77_86, 2003.

19. Bora S., and Dikenelli O., "Applying Feedback Control in Adaptive Replication in Fault Tolerant Multi-agent Organizations", Proc. ICSE'06 Fifth International Workshop on Software Engineering for Large-Scale Multi-Agent Systems (SELMAS'06), ACM. Shangai, China, 2006.

20. Bora S. and Dikenelli O.: Experience with Feedback Control Mechanisms in Self-replicating Multi-Agent Systems. CEEMAS 2007, 133-142, 2007.

21. Stollberg M.and Rhomberg F.: Survey on Goal-driven Architectures.Technical Report, 2006.

22. Ferber J. O. et al.: Agent/Group/Roles:Simulating with Organizations, Fourt International Workshop on Agent-Based Simulation, France, 2003.

23. Guerraoui R. and Schiper A.: Fault-Tolerance by Replication in Distributed Systems, Proc. Reliable Software Technologies, LNCS 1088, 1996.

24. SEAGENT, http://www.seagent.ege.edu.tr

25. Almeida A., Aknine S.,Briot J. P.: Dynamic resource allocation heuristics for providing fault tolerance in multi-agent systems. SAC 2008: 66-70.

26. Dastani M., Dignum V., Dignum F.: Role-Assignment in Open Agent Societies. In AAMAS'03, ACM 2003, Australia.

27. Powell D.: Delta-4: A generic architecture for dependable distributed computing. In Springer Verlag, 1991 